\title{
Carotid arterial hemodynamics in patients with essential hypertension of different dialectical types of traditional Chinese medicine
}

\author{
Peng Zhang ${ }^{1 \#}$, Yanjun Cui ${ }^{2 \#}$, Xuesong Yang" ${ }^{2 \#}$ Xiaoxia Zhao ${ }^{2}$, Meiying Jin ${ }^{3}$, Lei $\mathrm{Li}^{2}$ \\ ${ }^{1}$ First Clinical Medical College, Shandong University of Traditional Chinese Medicine, Jinan, China; ${ }^{2}$ Affiliated Hospital of Shandong University of \\ Traditional Chinese Medicine, Jinan, China; ${ }^{3}$ Yanzhou District Hospital of Traditional Chinese Medicine, Jining, China \\ Contributions: (I) Conception and design: P Zhang, L Li; (II) Administrative support: Y Cui; (III) Provision of study materials or patients: Y Cui, X \\ Yang; (IV) Collection and assembly of data: X Zhao; (V) Data analysis and interpretation: M Jin; (VI) Manuscript writing: All authors; (VII) Final \\ approval of manuscript: All authors. \\ \#These authors contributed equally to this work and should be regarded as co-first authors. \\ Correspondence to: Lei Li. Affiliated Hospital of Shandong University of Traditional Chinese Medicine, No. 16369, Jingshi Road, Jinan 250011, China. \\ Email: lilei19840127@163.com.
}

Background: To probe into the relationship between traditional Chinese medicine (TCM) different dialectical types of essential hypertension and carotid artery hemodynamics.

Methods: According to TCM, different dialectical types, 189 patients suffering from essential hypertension were divided into five type groups by two deputy chief physicians of TCM, according to TCM diagnosis and treatment guide for hypertension prepared by the Chinese society of TCM in 2011. A total of 189 patients were examined by transcranial color doppler (TCD) and compared with 47 normal subjects. The measuring target included middle cerebral artery (MCA) and anterior cerebral artery (ACA), recording the average blood flow velocity $(\mathrm{Vm})$ and vascular pulse index $(\mathrm{PI})$.

Results: In MCAVm and ACAVm, hyperpyrexia of liver syndrome showed unique advantages and positive correlation. In MCAPI and ACAPI, internal block because of phlegm dampness, stasis syndrome, deficiency of qi and blood syndrome, and internal block because of blood stasis syndrome had advantages and positive correlation, and the above three groups were not statistically significant.

Conclusions: Our study determined different dialectical types and objective material foundations of TCM in hypertension, correlating with carotid artery hemodynamics. Also, dialectical treatment would reduce the cerebrovascular accident.

Keywords: Essential hypertension; traditional Chinese medicine syndrome (TCM syndrome); different dialectical types; carotid artery hemodynamics; transcranial color doppler (TCD)

Submitted Sep 18, 2020. Accepted for publication Nov 04, 2020.

doi: 10.21037/apm-20-2060

View this article at: http://dx.doi.org/10.21037/apm-20-2060

\section{Introduction}

Hypertension is a global public health problem, causing many complications, especially cardiovascular events. The latest study (PURE) showed that hypertension was the largest risk factor, causing $22.3 \%$ of cardiovascular disease (CVD) cases and deaths (1). Strategies for early intervention, particularly for hypertension, are critical for reducing stroke morbidity and mortality (2). Its high incidence rate characterizes hypertension, high mortality rate, and high disability rate, which seriously endangers the health of our population $(3,4)$. Although western medicine has an apparent protective effect on blood pressure and target organs, it has adverse reactions and low compliance of patients (5). Combining Western Medicine or a single 
application of traditional Chinese medicine (TCM) can effectively reduce blood pressure, improve the quality of life of patients, an integral part of modern clinical treatment of essential hypertension $(6,7)$. As everyone knows, overweight, halophilic, lack of exercise, smoking, drinking, diabetes, hyperlipidemia, carotid plaque and so on are risk factors of hypertension. Antihypertensive treatment can be divided into non drug treatment and drug treatment. Non drug treatments include diet therapy (with emphasis on reducing salt intake), lifestyle changes (such as exercise, alcohol restriction, obesity reduction) and mechanical interventions such as continuous positive airway pressure (CPAP) for sleep apnea syndrome, renal artery intervention or adrenalectomy for secondary hypertension (8). The recent study (9) shows that cerebrovascular disease circulates through the whole pathological process of hypertension. Arterial stiffness as a risk factor for clinical hypertension is getting increased attention from scholars. Transcranial Doppler ultrasound can reveal the blood flow of intracranial vessels. Some scholars found that there was a correlation between carotid arterial hemodynamics in patients with essential hypertension of different dialectical types of TCM. But people's physique is also changing, with the change of time and lifestyle. In this case, it is of great significance to study whether the relationship has changed. The changes of hypertension patients with different TCM syndrome types are not completely consistent (8). The objective quantitative study of TCM syndrome differentiation on these differences will help to reveal the pathogenesis of hypertension and provide new ideas and directions for clinical treatment. We present the following article in accordance with the MDAR reporting checklist (available at http://dx.doi.org/10.21037/ apm-20-2060).

\section{Methods}

\section{General information}

Patients with essential hypertension treated in Affiliated Hospital of the Shandong University of Traditional Chinese Medicine, from January 2018 to June 2019, were considered the research objects.

All procedures performed in this study involving human participants were in accordance with the Declaration of Helsinki (as revised in 2013). Informed consent was taken from all the patients.

Inclusive criteria:

(I) The blood pressure of the patient who did not use antihypertensive drugs in the clinic was measured three times a day, systolic blood pressure (SBP) $\geq 140 \mathrm{mmHg}$ or diastolic blood (DBP) pressure $\geq 90 \mathrm{mmHg}$, or the patient who had a history of hypertension and used antihypertensive drugs at present was still diagnosed as hypertension, even if the blood pressure was lower than $140 / 90 \mathrm{mmHg}$, excluded all kinds of secondary hypertension and met with the diagnostic standard of essential hypertension (10);

(II) Age $\geq 18$ years old, gender unlimited;

(III) Patients know about the study and sign informed consent.

Exclusion criteria:

(I) Combined with heart, brain, kidney, or another primary organ disease;

(II) Combined with a malignant tumor, autoimmune, infectious disease, malignant tumor;

(III) Combined with severe arrhythmia, atrial fibrillation, atrial flutter, and other diseases;

(IV) Pregnancy and lactation;

(V) Combined with mental disorders and severe cognitive impairment;

(VI) Combined with intracranial vascular malformation; (VII) Took part in other clinical studies simultaneously.

One hundred eighty-nine patients were included, including 102 males and 87 females, aged 26-68 years. Also, the average age was $(52.38 \pm 9.85)$ years. Forty-seven subjects who received a physical examination in the Affiliated Hospital of the Shandong University of Traditional Chinese Medicine simultaneously were selected as the control group, including 26 males and 21 females, aged 2668 years, the average age was $(51.42 \pm 10.08)$ years. There was no significant difference in baseline data of gender and age between hypertension patients and the control group $(\mathrm{P}>0.05)$.

\section{TCM different dialectical types}

Two deputy chief physicians of TCM completed different dialectical types, according to the TCM diagnosis and treatment guide for bypertension prepared by the Chinese society of TCM in 2011:

(I) Hyperpyrexia of the liver syndrome (Ganhuo Shangyan, GHSY): the main symptoms are dizziness, swelling pain, red face, and red ears, irritability, and secondary symptoms are tinnitus, hypochondriac pain, bitter mouth, constipation, 
yellow tongue, yellow moss, and the number of pulse strings;

(II) Internal block because of blood stasis syndrome (Yuxue Neizu type, YXNZ): the main symptoms are headache, such as pricking, fixed pain, and secondary symptoms are chest distress, palpitation, numbness of hands and feet, especially at night, with a dark tongue and astringent pulse;

(III) Internal block because of phlegm dampness stasis syndrome (Tanshi Neizu type, TSNZ): the main symptom is head heavy as wrapping, and secondary symptoms are chest and epigastric fullness, dizziness and nausea, vomiting, phlegm and saliva, heavy sleepiness, less food and more sleep, greasy fur and slippery pulse;

(IV) Yin deficiency and Yang excess syndrome (Yinxu Yangkang, YXYK): the main symptoms are vertigo, tinnitus, soreness of loins and knees, five upset hot, and secondary symptoms are top-heavy, dry mouth and throat, dry and astringent eyes, red tongue, less moss, and fine veins;

(V) Deficiency of both qi and blood syndrome (Qixue Liangxu, QXLX): the main symptoms are vertigo, shortness of breath, dry mouth upset, and secondary symptoms are a white face, spontaneous sweating or night perspiration, palpitation, insomnia, narcolepsy, abdominal distention, loose stools, and other diseases, with a light tongue and a fine pulse.

\section{Transcranial color Doppler ultrasonography (TCD)}

All the subjects were examined using an experienced ultrasound doctor. We used the II TCD cerebral blood flow diagnostic instrument by EME company. The probe frequency was set to $2 \mathrm{MHz}$, the scanning range was middle cerebral artery (MCA), anterior cerebral artery (ACA). Bilateral middle cerebral artery (MCA) and anterior cerebral artery (ACA) were examined by temporal window and the average blood flow velocity (VM) and pulse index (PI) were also recorded.

\section{Statistical methods}

Spss23.0 was used for statistical data analysis. The mean and standard deviations expressed the measurement dates, and the count dates were expressed by rate or percentage. We used the single factor analysis of variance (One-way
ANOVA). Also, the pairwise comparison used the LSD $t$-test. Comparison of the differences between groups of date used $\chi^{2}$ test. $\mathrm{P}<0.05$ was considered statistically significant.

\section{Results}

\section{The composition ratio of TCM different dialectical types}

Among the 189 subjects, 34 were GHSY, accounting for 17.99\%; 26 were YXNZ, accounting for $13.76 \%$; 54 were TSNZ, accounting for $28.57 \%$; 39 were YXYK, accounting for $20.63 \%$; 36 were QXLX, accounting for $19.05 \%$.

\section{Comparison of TCD indexes in different dialectical types of TCM (Table 1)}

\section{MCAVm and ACAVm}

(I) GHSY type was the fastest of all groups $(\mathrm{P}<0.05)$;

(II) YXYK type was faster than the control group $(\mathrm{P}<0.05)$;

(III) The control group was faster than the other three groups: YXNZ type, TSNZ type, and QXLX type $(\mathrm{P}<0.05)$

\section{MCAPI and ACAPI}

(I) There was no significant difference between GHSY type and YXYK type with the control group $(\mathrm{P}>0.05)$;

(II) YXNZ type, TSNZ type, QXLX type were higher than those of the control group and other TCM types $(\mathrm{P}<0.05)$.

\section{Correlation between hypertension type and hemodynamics of intracranial artery (Table 2)}

\section{MCAVm}

(I) A positive correlation with GHSY $(\mathrm{P}<0.05)$;

(II) A negative correlation with YXNZ, TSNZ, and QXLX $(\mathrm{P}<0.05)$;

(III) No correlation with YXYK $(\mathrm{P}>0.05)$;

\section{ACAVm}

(I) A positive correlation with GHSY, YXYK $(\mathrm{P}<0.05)$;

(II) A negative correlation with YXNZ, TSNZ $(\mathrm{P}<0.05)$;

(III) No correlation with QXLX (P>0.05);

\section{MCAPI and ACAPI}

(I) A negative correlation with TSNZ, YXNZ, QXLX $(\mathrm{P}<0.05)$; 
Table 1 Comparison of TCD indexes in patients with different dialectical types

\begin{tabular}{|c|c|c|c|c|c|}
\hline Dialectical types & Number of cases & \multicolumn{2}{|c|}{ MCA } & \multicolumn{2}{|c|}{ ACA } \\
\hline GHSY & 34 & $102.47 \pm 21.85^{, I,\|,\|, I, V, V}$ & $0.94 \pm 0.13^{1, \mathrm{II}, \mathrm{V}}$ & $79.85 \pm 13.48^{1,\|,\|, \|, I, V, V}$ & $0.93 \pm 0.14^{1, \mathrm{II}, \mathrm{V}}$ \\
\hline YXNZ & 26 & $41.85 \pm 10.92^{1, \mathrm{II}, \mathrm{IV}}$ & $1.13 \pm 0.19^{\mathrm{l}, \mathrm{IV}}$ & $43.72 \pm 8.54^{1, \mathrm{II}, \mathrm{IV}, \mathrm{V}}$ & $1.22 \pm 0.19^{1, \mathrm{IV}}$ \\
\hline TSNZ & 54 & $48.63 \pm 10.28^{1, I V}$ & $1.08 \pm 0.13^{1, \mathrm{IV}}$ & $48.03 \pm 12.45^{\mathrm{I}, \mathrm{IV}, \mathrm{V}}$ & $1.18 \pm 0.21^{1, \mathrm{IV}}$ \\
\hline QXLX & 36 & $63.25 \pm 14.32^{\prime}$ & $1.12 \pm 0.15^{\prime}$ & $51.37 \pm 18.49$ & $1.22 \pm 0.14^{\prime}$ \\
\hline Control & 47 & $69.54 \pm 16.32$ & $0.93 \pm 0.16$ & $56.09 \pm 14.35$ & $0.95 \pm 0.18$ \\
\hline $\mathrm{F}$ & & 205.427 & 114.366 & 185.302 & 122.348 \\
\hline$P$ & & $<0.001$ & $<0.001$ & $<0.001$ & $<0.001$ \\
\hline
\end{tabular}

Compare with control: ' $\mathrm{P}<0.05$; compare with $\mathrm{YXNZ}$, " $\mathrm{P}<0.05$; compare with TSNZ, "II $\mathrm{P}<0.05$; compare with $\mathrm{YXYK}$, ${ }^{\mathrm{IV}} \mathrm{P}<0.05$; compare with $\mathrm{QXLX},{ }^{\vee} \mathrm{P}<0.05$. TCD, transcranial color Doppler; MCA, middle cerebral artery; ACA, anterior cerebral artery; PI, pulse index; GHSY, Ganhuo Shangyan; YXNZ, Yuxue Neizu type; TSNZ, Tanshi Neizu type; YXYK, Yinxu Yangkang; QXLX, Qixue Liangxu.

Table 2 Correlation between hypertension type and hemodynamics of intracranial artery

\begin{tabular}{lccccc}
\hline \multirow{2}{*}{$\begin{array}{l}\text { Dialectical } \\
\text { types }\end{array}$} & \multicolumn{2}{c}{ MCA } & & \multicolumn{2}{c}{ ACA } \\
\cline { 2 - 3 } \cline { 5 - 6 } GHSY & $0.502^{\prime}$ & 0.113 & & $0.433^{\prime}$ & -0.063 \\
YXNZ & $-0.382^{\prime}$ & $0.328^{\prime}$ & & $-0.311^{\prime}$ & $0.486^{\prime}$ \\
TSNZ & $-0.418^{\prime}$ & $0.315^{\prime}$ & & $-0.286^{\prime}$ & $0.433^{\prime}$ \\
YXYK & 0.173 & -0.083 & & $0.273^{\prime}$ & -0.092 \\
QXLX & $-0.274^{\prime}$ & $-0.271^{\prime}$ & & 0.102 & $0.305^{\prime}$ \\
\hline
\end{tabular}

'P<0.05. TCD, transcranial color Doppler; MCA, middle cerebral artery; ACA, anterior cerebral artery; PI, pulse index; GHSY, Ganhuo Shangyan; YXNZ, Yuxue Neizu type; TSNZ, Tanshi Neizu type; YXYK, Yinxu Yangkang; QXLX, Qixue Liangxu

(II) No correlation with GHSY, YXYK $(\mathrm{P}>0.05)$;

\section{Discussion}

Essential hypertension belongs to "vertigo" and "headache" of TCM, which has a unique advantage in the diagnosis and treatment of essential hypertension. These two studies $(11,12)$ show that combining TCM and exercise therapy has a positive effect on controlling blood level and improving target organ damage. According to TCM, the disease is located in the brain, and its pathological changes are in the liver, spleen, and kidney, no more glory in the brain due to deficiency of Qi and blood, Yin and essence, or the brain is blinded by phlegm and turbid block, or the liver Yang turns into the wind, which interferes with the clearing up and causes vertigo.

Craniocerebral and intracranial blood vessels are the target organs and blood vessels most easily and earliest affected by hypertension. A recent study shows that when the systemic signs and clinical symptoms are not apparent in the early stage of hypertension, the changes in cerebral hemodynamics can appear. Early recognition of the changes in cerebral hemodynamics in patients with hypertension has a suggestive role in early diagnosis (13).

\section{Comparison of TCD indexes in different TCM dialectical types}

In this research, MCAVm and ACAVm of GHSY were higher than the control group and other dialectical types. The reasons are as follows: The five elements of the liver belong to wood, which is active in ascending, belongs to Yin in physique, and Yang in function. Liver fire is susceptible to hyperactivity due to long-term melancholy and anger, upward attacking orifices in the head, causing headaches, dizziness, dizziness, and other symptoms (14). The phenomenon also leads to the acceleration of cerebral blood flow due to the blood rushing up. Therefore, it was observed that the speed of MCAVm and ACAVm increased significantly, and the symptoms of dizziness, swelling, pain, blushing are all related to this.

MCAVm and ACAVm of YXYK were higher than 
the standard healthy control group and the other three syndrome types, lower than GHSY. Hyperactivity of liver Yang can cause headaches and dizziness due to deficiency of liver yin, which is contrary to upward, leading to upward blood flow. Deficiency of Yin, not restraining Yang, is the asthenic symptoms and substance; therefore, the acceleration of blood flow is not as satisfactory as GHSY.

MCAVm and ACAVm of TSNZ, YXNZ, and QXLX were lower than the control group. The reasons are as follows: Phlegm and turbid phlegm block the Qi mechanism, which is not smooth, resulting in stagnant blood flow (15). A recent study (16) showed that YXNZ exhibited vastly increased RBC aggregation and stagnant blood flow. Deficiency of Qixue and weakness of agitation lead to low blood circulation and slowdown of blood flow.

A recent study (17) has shown that PI can reflect the pulsation and resistance of intracranial vessels, and it is often used to reflect the compliance and elasticity of arteries. MCAPI and ACAPI of YXNZ were higher than the standard healthy control group and other syndrome types, due to increased RBC aggregation and stagnant blood flow.

\section{Correlation between hypertension type and bemodynamics of intracranial artery}

\section{MCAVm and ACAVm}

GHSY type and YXYK type belong to the surplus of Yang Qi. The difference between them was a reality (GHSY) and the deficiency (YXYK). The results of MCAVm and ACAVm confirmed the active, positive Yang can accelerate blood flow, which is also consistent with the theory of Traditional Chinese Medicine, "forgetting blood heat".

\section{MCA and ACAPI}

TSNZ type and YXNZ type are tangible objects belonging to Yin, which can hinder the movement of Qi and blood. QXLX type often leads to qi stagnation, blood stasis, or phlegm dampness, affecting the operation of Qi and blood.

To sum up, the results of this study showed that there is a specific relationship between TCM syndrome differentiation and typing of essential hypertension and artery hemodynamics. However, there were few patients in this study, which could not cover all syndromes of essential hypertension. The results of this study still need to be included in the multi-center study of more cases for further demonstration.

\section{Acknowledgments}

Funding: None.

\section{Footnote}

Reporting Checklist: The authors have completed the MDAR reporting checklist. Available at http://dx.doi.org/10.21037/ apm-20-2060

Data Sharing Statement: Available at http://dx.doi. org/10.21037/apm-20-2060

Conflicts of Interest: All authors have completed the ICMJE uniform disclosure form (available at http://dx.doi. org/10.21037/apm-20-2060). The authors have no conflicts of interest to declare.

Ethical Statement: The authors are accountable for all aspects of the work in ensuring that questions related to the accuracy or integrity of any part of the work are appropriately investigated and resolved. All procedures performed in this study involving human participants were in accordance with the Declaration of Helsinki (as revised in 2013). Informed consent was taken from all the patients.

Open Access Statement: This is an Open Access article distributed in accordance with the Creative Commons Attribution-NonCommercial-NoDerivs 4.0 International License (CC BY-NC-ND 4.0), which permits the noncommercial replication and distribution of the article with the strict proviso that no changes or edits are made and the original work is properly cited (including links to both the formal publication through the relevant DOI and the license). See: https://creativecommons.org/licenses/by-nc-nd/4.0/.

\section{References}

1. Yusuf $\mathrm{S}$, Joseph $\mathrm{P}$, Rangarajan $\mathrm{S}$, et al. Modifiable risk factors, cardiovascular disease, and mortality in 155722 individuals from 21 high-income, middle-income, and low-income countries (PURE): a prospective cohort study. Lancet 2020;395:795-808.

2. Furie K. Epidemiology and Primary Prevention of Stroke. Continuum (Minneap Minn) 2020;26:260-7.

3. Zhang L, Wang Z, Wang X, et al. Prevalence of overweight and obesity in China: Results from a crosssectional study of 441 thousand adults, 2012-2015. Obes 
Res Clin Pract 2020;14:119-26.

4. Yang XH, Zhang WX, Shuai $W$, et al. Clinical diagnosis rate of hypertension newly detected in epidemiological survey. Chinese J Hypertension 2018;26:1026-9.

5. Fang ZY. Clinical review of traditional Chinese medicine in the treatment of hypertension. Jiangsu Traditional Chinese Medicine 2019;51:1-6.

6. Lin XF. Clinical observation of Tianma Gouteng Decoction Combined with captopril in the treatment of hypertension with hyperactivity of liver Yang. Journal of Liaoning Medical College 2017;38:25-7.

7. Li MH, Chen L, Liang H. Analysis of curative effect and effective components of Banxia Baizhu Tianma decoction combined with Wendan Decoction on hypertension. Shanxi Medical Journal 2018;(5):555-7.

8. Vitali C, Bombardieri S, Jonsson R, et al. Classification criteria for Sjögren's syndrome: a revised version of the European criteria proposed by the American-European Consensus Group. Ann Rheum Dis 2002;61:554-8.

9. Deng R, Liu Y, Song YY, et al. A comparative study of transcranial Doppler dynamic monitoring and CT dynamic scanning after minimally invasive drainage of cerebral hemorrhage. Chinese J Physicians 2017;19:450-2.

10. Shang QQ, Wang L, Wang YQ, et al. Research progress on etiology, pathogenesis and syndrome of essential hypertension in traditional Chinese medicine. Hebei Traditional Chinese Medicine 2017;39:138-42.

11. Liu DJ, Liu ZJ, Jiang KL, et al. Based on data mining to explore the law of Xian Shaoxiang's syndrome differentiation and medication in the treatment of hypertension and headache. Journal of Guangzhou

Cite this article as: Zhang P, Cui Y, Yang X, Zhao X, Jin M, Li L. Carotid arterial hemodynamics in patients with essential hypertension of different dialectical types of traditional Chinese medicine. Ann Palliat Med 2020;9(6):4097-4102. doi: 10.21037/ apm-20-2060
University of Traditional Chinese Medicine 2018;35:558-61.

12. Zheng XY. Guiding principles for clinical research of new Chinese medicine. Beijing: China Medical Science and Technology Press, 2002:73-7.

13. Wang T, Li YH, Guo XH, et al. Application of threedimensional quasi continuous arterial spin labeled perfusion imaging in quantitative changes of cerebral hemodynamics in patients with hypertension. Journal of Chinese Academy of Medical Sciences 2017;(4):477-84.

14. Wang JM, Song XB, Liu N, et al. Clinical study on the treatment of vertigo (hypertension) caused by liver fire by "treating both the upper and the lower" method. World Journal of Integrated Traditional and Western Medicine 2017;12:1264-6.

15. Li WF, Chen SQ. Logistic analysis of TCM syndrome type risk factors of chronic heart failure caused by hypertension. World TCM 2019;14:1330-4.

16. Zhong WR, Ou WY, Peng CJ. The effect of traditional Chinese medicine for promoting qi and removing stasis combined with western medicine on the treatment of primary hypertension with blood stasis syndrome and the effect on the level of Hcy and $25(\mathrm{OH}) \mathrm{d}$. Journal of Cardio Cerebrovascular Diseases with Integrated Chinese and Western Medicine 2018;16:2379-82.

17. Li R, Zhao PF, Zhang XJ, et al. Based on CTA and ultrasound to explore the relationship between intracranial carotid stenosis and ocular artery blood flow. J Clin Exp Med 2017;16:1267-71.

(English Language Editor: J. Chapnick) 